\title{
Ductility by shear band delocalization in the nano- layer of gradient structure
}

\author{
Fuping Yuan, Dingshun Yan, Jiangda Sun, Lingling Zhou, Yuntian Zhu \& \\ Xiaolei Wu
}

To cite this article: Fuping Yuan, Dingshun Yan, Jiangda Sun, Lingling Zhou, Yuntian Zhu \& Xiaolei Wu (2019) Ductility by shear band delocalization in the nano-layer of gradient structure, Materials Research Letters, 7:1, 12-17, DOI: 10.1080/21663831.2018.1546238

To link to this article: https://doi.org/10.1080/21663831.2018.1546238
(C) 2018 The Author(s). Published by Informa UK Limited, trading as Taylor \& Francis Group

\section{$+\quad$ View supplementary material $匚$}

\section{曲 Published online: 19 Nov 2018.}

Submit your article to this journal $\widetilde{ }$

Џ Article views: 846

\section{View Crossmark data ๔}




\title{
Ductility by shear band delocalization in the nano-layer of gradient structure
}

\author{
Fuping Yuan ${ }^{a, b}$, Dingshun Yan ${ }^{a}$, Jiangda Sun ${ }^{a}$, Lingling Zhou ${ }^{a}$, Yuntian Zhu ${ }^{c, d}$ and Xiaolei Wu ${ }^{a, b}$ \\ ${ }^{a}$ State Key Laboratory of Nonlinear Mechanics, Chinese Academy of Science, Institute of Mechanics, Beijing, People's Republic of China; b School \\ of Engineering Science, University of Chinese Academy of Sciences, Beijing, People's Republic of China; ${ }^{C}$ Nano and Heterostructural Materials \\ Center, School of Materials Science and Engineering, Nanjing University of Science and Technology, Nanjing, People's Republic of China; \\ ${ }^{\mathrm{d} D e p a r t m e n t}$ of Materials Science and Engineering, North Carolina State University, Raleigh, NC, USA
}

\section{ABSTRACT}

Nanostructured (NS) metals typically fail soon after yielding, starting with the formation of narrow shear bands. Here we report the observation of shear band delocalization in gradient metals. Shear bands were nucleated and delocalized in the NS layers by propagating along the gage length soon after yielding, converting the shear band into a localized strain zone (LSZ). Synergistic work hardening was developed in the LSZ by regaining dislocation hardening capability, and by back-stress hardening from the strain gradients in the axial and depth directions, which helped with enhancing global ductility.

\section{ARTICLE HISTORY}

Received 28 August 2018

\section{KEYWORDS}

Gradient structure; ductility; shear band; strain delocalization; back-stress hardening
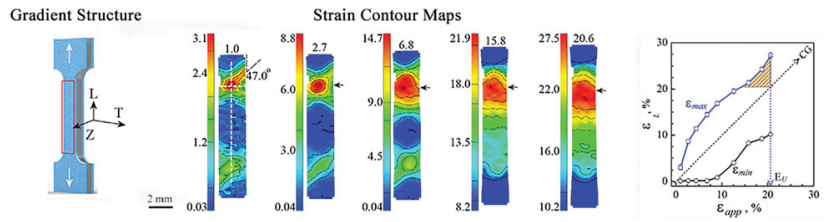

Nanostructured (NS) metals feature high strength [1-8], but typically have low ductility [1,9-12]. When stretched alone, NS metals intrinsically tend to develop shear bands soon after yielding, which propagate quickly through the cross-section [9-12], ending uniform elongation. This is because NS metals have low strain hardening capability [1,9-12]. Strategies for improving ductility [9-19] are mostly related to the recovery of intra-granular dislocation-mediated plasticity [5,10-14], which is, however, limited by either the small grain sizes or initial nearsaturation dislocation density $[2,3,9,10]$, both of which limit dislocation accumulation capability during tensile testing [9-11].

Ductility is generally defined as uniform elongation in the specimen gage length during tensile testing [20]. It can be determined by the Considère criterion [21], $(\mathrm{d} \sigma / \mathrm{d} \varepsilon) / \sigma \geq 1$, where $\sigma$ is true stress and $\epsilon$ true strain, or by the Hart's criterion [20], $(\mathrm{d} \sigma / \mathrm{d} \varepsilon) / \sigma+m \geq$
1 ,where $m$ is the strain rate sensitivity. Neither criterion literally specifies whether homogeneous strain in the gage section is a must for ductility, which raises a question: is it possible to maintain high ductility if strain is not uniform?

We investigated this issue using a gradient structured (GS) interstitial-free (IF) steel, consisting of NS surface layers with a continuous increase in grain sizes along the depth to the central coarse-grained (CG) layer [11,12]. It is found that shear bands were formed in the NS layers at a very early stage during tensile testing, as NS metals typically do. Unexpectedly, the shear bands became stabilized due to strain gradient and propagated slowly along the gage length to become a localized strain zone (LSZ), which produced synergistic strain hardening to help with retaining ductility. In other words, the shear bands helped with retaining ductility, contrary to our conventional understanding of strain localization [22-24].

CONTACT Yuntian Zhu ytzhu@ncsu.edu N Nano and Heterostructural Materials Center, School of Materials Science and Engineering, Nanjing University of Science and Technology, 200 Xiaolingwei, Nanjing 210094, People's Republic of China; Department of Materials Science and Engineering, North Carolina State University, Campus Box 7919, Raleigh, NC 27695, USA; Xiaolei Wu xlwu@imech.ac.cn State Key Laboratory of Nonlinear Mechanics, Chinese Academy of Science, Institute of Mechanics, 15 Beisihuan West Road, Beijing 100190, People's Republic of China; School of Engineering Science, University of Chinese Academy of Sciences, 19A Yuquan Road, Beijing 100049, People's Republic of China

(-) Supplemental data for this article can be accessed here. https://doi.org/10.1080/21663831.2018.1546238 
CG IF steel plate of $1 \mathrm{~mm}$ thick with a mean grain size of $26 \mu \mathrm{m}$ was used as the initial material. Gradient structure (GS) was prepared using surface mechanical attrition treatment (SMAT) technique [25]. The NS layer is $\sim 40 \mu \mathrm{m}$ thick, with a mean grain size of $200 \mathrm{~nm}$.

The tensile samples were dog-bone shaped with the gauge section of $1 \mathrm{~mm} \times 2 \mathrm{~mm} \times 10 \mathrm{~mm}$. Uniaxial tensile tests were conducted at a strain rate $\left(\dot{\varepsilon}_{\text {app }}\right)$ of $8 \times 10^{-4} \mathrm{~s}^{-1}$ under room temperature. Digital image correlation (DIC) imaging was performed on the top NS surface layer (see Supplementary Material). Microstructures, texture, and Vickers micro-hardness $\left(H_{\mathrm{V}}\right)$ were characterized on samples subjected to various tensile strains. $H_{\mathrm{V}}$ was measured with load of $25 \mathrm{~g}$ and dwell period of $15 \mathrm{~s}$ on NS surface after grinding off surface roughness by $\sim 10 \mu \mathrm{m}$ deep. Focused ion beam was used to cut the cross-sectional transmission electron microscopy (TEM) samples precisely in the shear band and LSZ in the NS layer at varying tensile strains according to the DIC image.

Figure 1(a) shows the microstructure of the central CG layer with an average grain size of $26 \mu \mathrm{m}$, and Figure 1(b) shows the nanostructure in the NS surface layer at $\sim 40 \mu \mathrm{m}$ depth, which reveals entangled dislocations in grain interiors and the average grain size is $200 \mathrm{~nm}$, typical of severely deformed metals $[3,4,26]$. The corresponding $H_{\mathrm{V}}$ gradient is visible from the surface to the center (Figure 1(c)).

Figure 2(a) shows the true stress-strain $(\sigma-\epsilon)$ curves of GS and CG samples. The GS sample shows uniform elongation $\left(E_{\mathrm{U}}\right)$ of $20.6 \%$, which retained about $80 \%$ of that $(26 \%)$ of the CG sample, while doubled yield strength. Figure 2(b) shows a set of typical contour maps showing longitudinal (axial) strain $\left(\varepsilon_{\mathrm{L}}, \%\right)$ distribution at varying applied tensile strains $\left(\varepsilon_{\text {app }}, \%\right)$. At $\varepsilon_{\text {app }}=1 \%$, two shear bands crossing each other were formed in the upper part of the sample. The shear bands are measured at $\sim 45^{\circ}$ to tensile axis, with the orientation of maximum resolved shear stress. Thus, the plastic response in NS layer begins with the nucleation of shear bands. These two shear bands propagated downward with increasing $\varepsilon_{\text {app }}$ along the gauge length and continually broadens to form an LSZ, see maps with increasing $\varepsilon_{\text {app }}$ from $2.7 \%$ to $15.8 \%$ until necking at $\varepsilon_{\text {app }}=20.6 \%\left(E_{\mathrm{U}}\right)$ (Figure 2(a)). A weaker LSZ is also visible in the lower part but fail to propagate much. In every sample tested, there is only one dominant LSZ, which led to the failure of whole sample.

The LSZ accumulated plastic strains continuously. Figure 2(c) shows heterogeneous $\varepsilon_{\mathrm{L}}$ at varying $\varepsilon_{\text {app }}$ as a result of the propagating LSZ. $\varepsilon_{\mathrm{L}}$ was measured along a longitudinal line which goes through the maximum $\varepsilon_{\mathrm{L}}\left(\varepsilon_{\max }\right)$ in each contour, e.g. white line at $\varepsilon_{\text {app }}=1 \%$ in Figure 2(b). The LSZ is defined, here, as that with $\varepsilon_{\mathrm{L}}>\varepsilon_{\text {app }}$, e.g. the segment bounded by two $\times$ marks in the curve at $\varepsilon_{\text {app }}$ of $15.8 \%$. Figure 2(d) shows the evolution of $\varepsilon_{\max }$ and minimum $\varepsilon_{\mathrm{L}}\left(\varepsilon_{\min }\right)$ in NS layer. Note that $\varepsilon_{\max }$ is always in the center of the LSZ. Figure 2(e) shows the evolution of average $\varepsilon_{\mathrm{L}}$ in LSZ, $\bar{\varepsilon}_{\text {LSZ }}$. Figure $2(\mathrm{f}$ ) shows the axial maximum strain rate $\dot{\varepsilon}_{\mathrm{L}}\left(\dot{\varepsilon}_{\max }\right)$ in NS layer calculated by $\dot{\varepsilon}_{\mathrm{L}}=\partial \varepsilon / \partial t$. $\dot{\varepsilon}_{\max }$ is found always at the propagating front of the LSZ and can be used as an indicator for the propagating rate of LSZ.

From Figure 2(b-f), several features of the plastic deformation can be drawn. Firstly, the shear band/LSZ sustained more strain in its interior than outside with increasing $\varepsilon_{\text {app }}$ (Figure 2(c,d)), typical of strain localization. The left and right peaks of $\varepsilon_{\mathrm{L}}$, e.g. at $\varepsilon_{\text {app }} \leq 6.8 \%$ (Figure 2(c)), represent the upper and the lower shear bands, while the latter disappeared at $\varepsilon_{\text {app }}=15.8 \%$. Secondly, $\varepsilon_{\mathrm{L}}$ is not uniform in the NS layer during the whole testing (Figure $2(\mathrm{~b}-\mathrm{d})$ ). Moreover, $\varepsilon_{\max }$ in shear band/LSZ is larger than $\varepsilon_{\text {app }}$, even than $E_{\mathrm{U}}$ (shadowed area in Figure 2(d)). In contrast, $\varepsilon_{\mathrm{L}}$ is equal to $\varepsilon_{\text {app }}$ in CG (see Figure S1 in the Supplementary Material), as represented by the diagonal dotted line, due to uniform deformation in the stable CG layer before necking. Thirdly, the ratio of $\bar{\varepsilon}_{\text {LSZ }} / \varepsilon_{\text {app }}$ in the LSZ (Figure 2(e)) can be seen as an indicator on the severity of strain localization. As seen, strain localization started at low
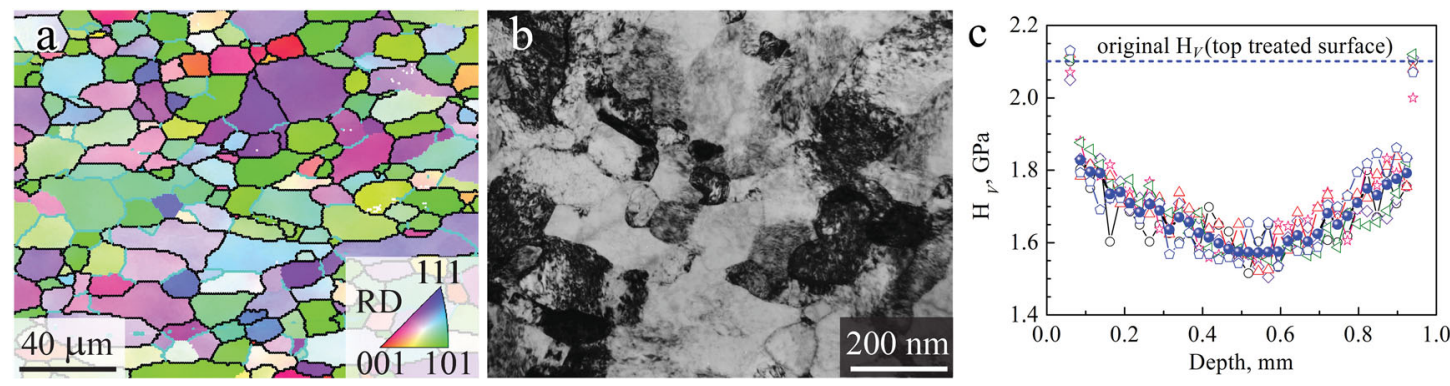

Figure 1. Microstructure and Micro-hardness $\left(H_{\mathrm{V}}\right)$ in GS IF steel. (a) Electron back-scatter diffraction image showing coarse-grains in the central CG layer. (b) TEM image showing nano-grains with high density of dislocations at $\sim 40 \mu \mathrm{m}$ depth in NS layer. (c) $\mathrm{H}_{V}$ gradient along the depth. 

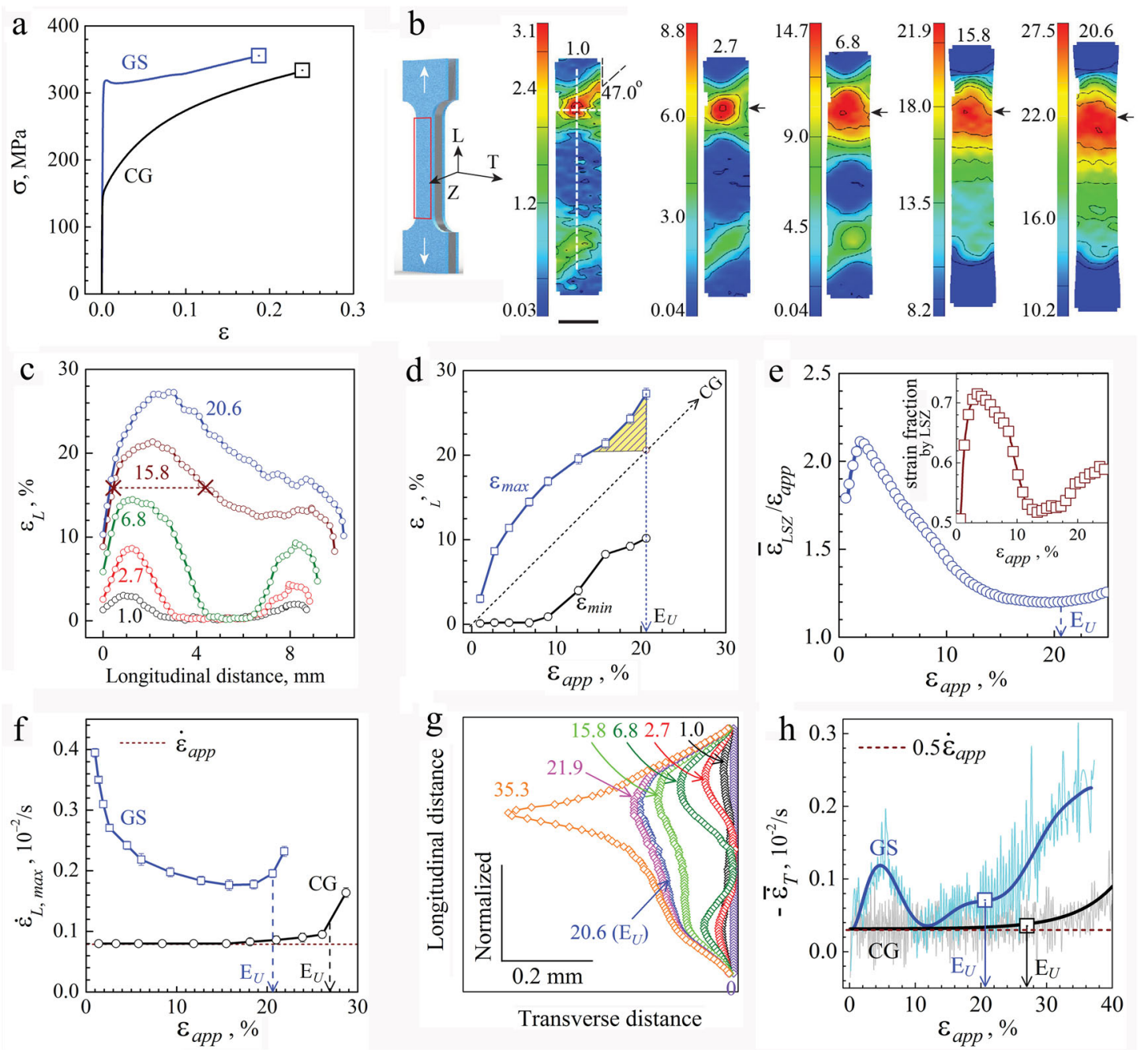

Figure 2. Strain localization and delocalization in LSZ. (a) Tensile true stress-strain $(\sigma-\epsilon)$ curves measured by DIC testing. GS: gradientstructured sample. CG: coarse-grained sample. Square on each curve: uniform elongation $\left(E_{\mathrm{U}}\right)$. (b) Contour maps of axial strain ( $\left.\varepsilon_{\mathrm{L}}\right)$ on NS surface vs. applied tensile strain $\left(\varepsilon_{\text {app }}\right)$. Surface area of the gauge section: $2 \times 10 \mathrm{~mm}^{2}$. Number above each map: $\varepsilon_{\text {app }}(\%)$. Scale bar (color): range of $\varepsilon_{\mathrm{L}}$ in whole contour, with maximum (defined as $\varepsilon_{\max }$ ) (top number) and minimum $\varepsilon_{\mathrm{L}}$ (defined as $\varepsilon_{\min }$ ). Scale bar (horizontal): $2 \mathrm{~mm}$. (c) Distribution of $\varepsilon_{\mathrm{L}}$ at varying $\varepsilon_{\text {app }}$ along axial position, e.g. white vertical line in the first contour in (d). (d) Maximum and minimum $\varepsilon_{\mathrm{L}}\left(\varepsilon_{\max }\right.$ and $\left.\varepsilon_{\min }\right)$ vs. $\varepsilon_{\text {app }}$. Diagonal dashed line: uniform $\varepsilon_{\mathrm{L}}$ of CG sample. (e) $\bar{\varepsilon}_{\mathrm{LSZ}} / \varepsilon_{\mathrm{app}}$ vs. $\varepsilon_{\text {app. }} \cdot \bar{\varepsilon}_{\mathrm{LSZ}}$ : axial average strain in LSZ. Inset: Strain fraction supplied by the LSZ at varying $\varepsilon_{\mathrm{L}}$. (f) Axial maximum strain rate $\left(\dot{\varepsilon}_{\mathrm{L}}=\mathrm{d} \varepsilon_{\mathrm{L}} / \mathrm{d} L\right)$ at the propagating front of the LSZ vs. $\varepsilon_{\text {app }}$. (g) Profiles of lateral shrinkages in (b) at varying $\varepsilon_{\text {app }}$ (number, \%). (h) Average lateral shrinkage rate at the upper LSZ vs. $\varepsilon$ app.

$\varepsilon_{\text {app }}$ of $\sim 1 \%$, reached the maximum at $\varepsilon_{\text {app }}=2.0 \%$, and then decreased until the end of uniform elongation $\left(E_{\mathrm{U}}\right)$. Fourthly, $\dot{\varepsilon}_{\max }$ started about one order of magnitude larger than $\dot{\varepsilon}_{\text {app }}=8 \times 10^{-4} \mathrm{~s}^{-1}$ (Figure 2(f)) and dropped monotonously. In other words, the shear band/LSZ propagated fast initially, but slowed down later. In contrast, the CG sample shows a nearly constant $\dot{\varepsilon}_{\mathrm{L}}$ until necking (Figure 2(f) and Figure S1 in SI). Most importantly, the applied strain is mostly sustained in the LSZ. The deformation fraction supplied by LSZ is calculated by $\bar{\varepsilon}_{\text {LSZ }} \times$ area ofLSZ) $/\left(\varepsilon_{\text {app }} \times\right.$ gauge area $)$. As seen in the inset of Figure 2(e), the LSZ accommodated the majority of the applied tensile strains.
Figure $2(\mathrm{~g})$ shows the changing profiles of lateral shrinkage at varying $\varepsilon_{\text {app }}$ by subtracting local width from the largest real-time width. The location of localized shrinkage coincides with that of the LSZ, see the arrows in contour maps at varying $\varepsilon_{\text {app }}$ in Figure 2(b). In other words, the LSZ triggered localized lateral shrinkage at the very early stage. This is unexpected because the sample was still globally stable with strong strain hardening. Figure 2(h) shows the average lateral shrinkage rate $\left(\dot{\varepsilon}_{\mathrm{T}}\right)$. It reached the peak at $\varepsilon_{\text {app }} \sim 5 \%$, decreased subsequently, and increased again until global necking $\left(E_{\mathrm{U}}\right)$. In contrast, the CG sample has constant $\dot{\varepsilon}_{\mathrm{T}}$, equal to half of $\dot{\varepsilon}_{\text {app }}$, typical of uniform plastic deformation. 
The heterogeneous $\varepsilon_{\mathrm{L}}$ (Figure 2(c)) caused axial strain gradient, $\mathrm{d} \varepsilon_{\mathrm{L}} / \mathrm{d} L$, near the LSZ boundaries in the NS layer. The maximum strain gradient lies always at the front of propagating LSZ, and increased with $\varepsilon_{\text {app }}$ (Figure 3(a)). Strain softening occurred in the center of the LSZ at the early stage of shear band formation (Figure 3(b)), showing dramatic drop of $H_{\mathrm{V}} . H_{\mathrm{V}}$ increased later with increasing $\varepsilon_{\text {app }}$ from $6.8 \%$ to $15.8 \%$, indicating that it recovered some strain hardening capability (Figure 3(c)).

Strain gradient arises from mechanical incompatibility. The propagating front of the LSZ demarcates its boundary (Figure 3(b)). As shown, there is a steep strength gradient at the LSZ boundary, which will lead to strain gradient during tensile deformation. Geometrically necessary dislocations need to be produced to facilitate the strain gradient [27-29], which will produce strong back-stress hardening [14,30-36]. The back-stress hardening will impede the axial rapid propagation of the LSZ, which helps with the stabilization and delocalization of the shear band/the LSZ, leading to the drop of $\dot{\varepsilon}_{\mathrm{L}}$ with $\varepsilon_{\mathrm{app}}$ (Figure $2(\mathrm{~g})$ ).

The initial drop and later rise of $H_{\mathrm{V}}$ in the center of the LSZ (Figure 3(b,c)) indicate the recovery of dislocation strain hardening. Figure 4(a) shows a weak compression shear texture with (110) orientation parallel to compressive axis due to SMAT before tensile testing (a-1), which evolved later into strong tensile textures with (110) orientation parallel to tensile axis at $\varepsilon_{\text {app }}$ of $15.8 \%$. The tensile texture strength is especially strong at the center of the LSZ, as indicated in Figure 4(a3 ), as compared with locations outside of the LSZ (as indicated in Figure 4(a-2). This indicates strong dislocation activities in nanograins in the LSZ during tensile deformation.

The dislocation activity is corroborated by TEM observations. At $\varepsilon_{\text {app }}$ of $4 \%$ (Figure $4(\mathrm{~b})$ ), the dislocations are hardly seen inside most grains in the center of the
LSZ, in contrast to high density of dislocations before tensile testing (Figure 1(b)). Inset at the top left corner of Figure 4(b) reveals dislocation debris in a few grains. This indicates dismantlement of original dislocation substructure due to the change of strain path and stress state $[12,37]$. This is the reason of the observed strain softening in the shear band/LSZ (Figure 3(b)). Further straining led to the formation of new dislocation networks near grain boundaries, as shown in Figure 4(c) $\left(\varepsilon_{\text {app }}=\right.$ $20.6 \%)$. This is caused by the complex stress state $[12,37]$ in the LSZ, where multiple slip systems are activated, which in turn forms new dislocation entanglements and accumulation (see the inset). The change in dislocation density coincides with that of $H_{\mathrm{V}}$ (Figure 3(c)), suggesting their close relationship. Furthermore, the mean grain size is maintained close to $\sim 200 \mathrm{~nm}$ in the LSZ during the tensile testing, indicating no grain growth in the LSZ.

It should be noted that initial softening and recovered hardening observed in the LSZ has some similarity and difference from the reported hardness fluctuation observed during the severe plastic deformation of a nanocrystalline $\mathrm{Ni}-\mathrm{Fe}$ alloy [38]. Although both cases were linked to dislocation density change, the initial softening and recovered hardening in the LSZ was caused by the change of strain path, while the latter was observed during severe straining in the strain direction.

The NS layer and central CG layer in the GS sample were subjected to the same amount applied tensile strain. The NS layer deformed by propagating shear bands (Figure 2(b,c)), while the CG layer deformed uniformly. Strain delocalization in the shear bands/LSZ helped with retaining ductility in NS layer. In contrast, shear bands would have failed homogeneous NS metals quickly $[2,3,11,12]$. The LSZ regained strain hardening capability after the initial softening (Figure 4(c)), a phenomenon that would rarely occur in homogeneous NS metals [38]. Both forest dislocation hardening and
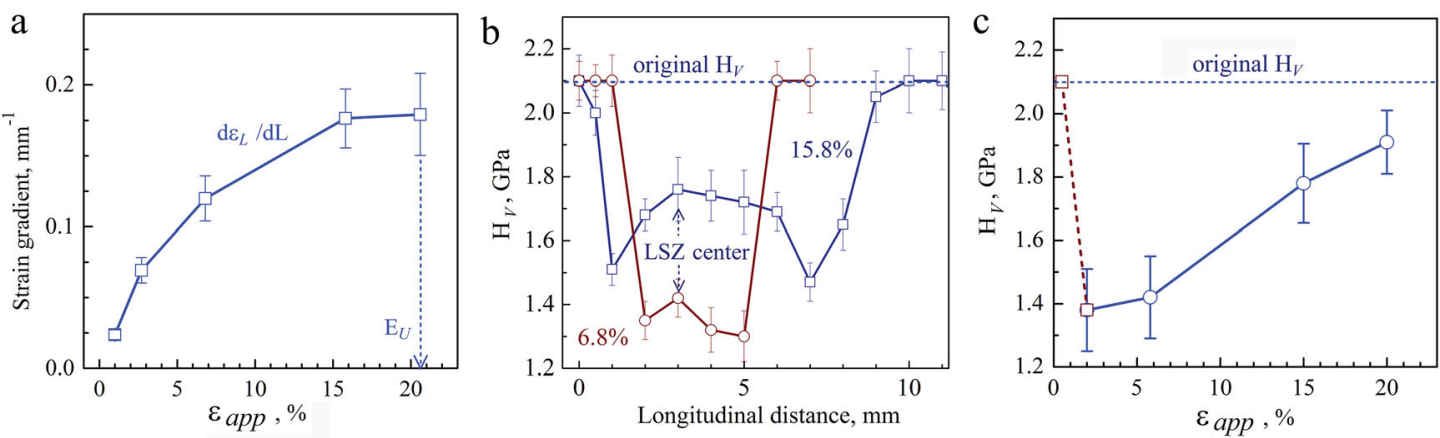

Figure 3. Evolution of both maximum axial strain gradient $\left(\Psi_{\max }\right)$ and microhardness $\left(H_{\mathrm{V}}\right)$. (a) $\Psi_{\max }$ at the front of the propagating LSZ vs. $\varepsilon_{\text {app }}$. (b) $H_{\mathrm{V}}$ variation along axial LSZ at $\varepsilon_{\mathrm{app}}$ of $6.8 \%$ and $15.8 \%$. (c) Change of $H_{\mathrm{V}}$ at the center of the LSZ vs. $\varepsilon_{\mathrm{app}}$. 
a
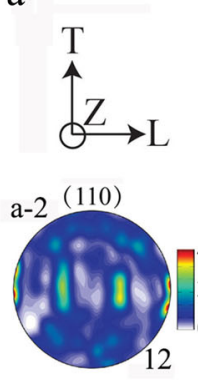

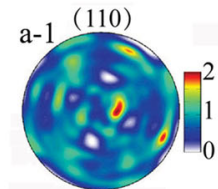

a-3 (110)
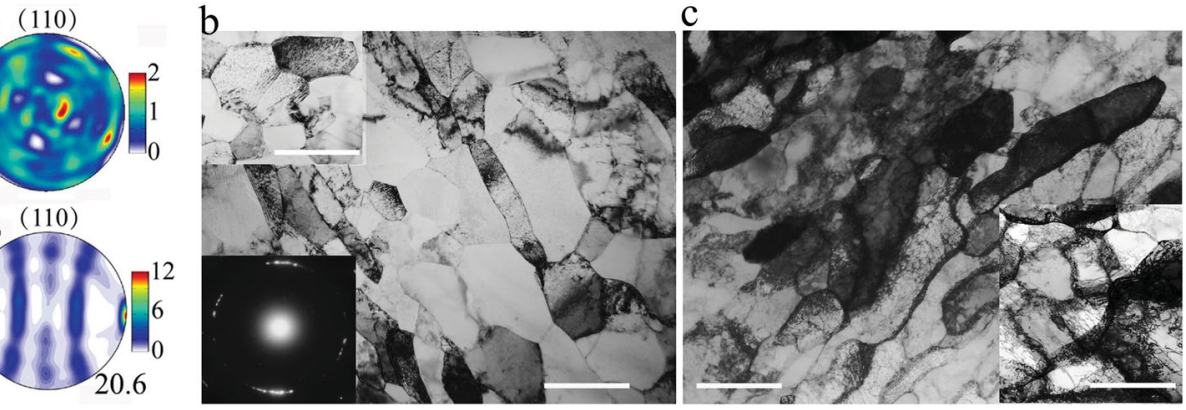

Figure 4. Evolution of texture and dislocation density. (a) a-1: initial compressive texture. a- 2 and a-3 at $\varepsilon_{\text {app }}=15.8 \%$ : tensile texture outside and at the center of LSZ. Number: local axial strain. (b) and (c) TEM micrographs showing dislocation density in the LSZ at $\varepsilon_{\text {app }}$ of $4 \%$ and $15.8 \%$, respectively. Inset in (b) (lower left): selected area electron diffraction pattern in the NC layer. Inset in (b) (upper left): dislocation debris. Inset in (c): newly-generated dislocations at grain boundaries. Scale bars: $200 \mathrm{~nm}$.

back-stress hardening occurred to stabilize the shear band [14,33], making it possible for its delocalization. The propagation of shear banding into the sample depth is deterred because the underneath CG layer is stable. This induces strain gradient in the depth direction and back-stress hardening to prevent the LSZ from propagating into the depth $[12,31,34]$.

The strategy of utilizing the shear band delocalization to develop synergistic work hardening for improving the ductility is expected also applicable to other heterostructured metals consisting of NS and CG domains. Another way to delocalize strains caused by shear bands is to develop high-density of them all over the NS domains so that no individual shear band will fail the specimen. Similar synergistic work hardening as discussed above should also work in this situation to improve ductility. In fact, such types of LSZs have been observed in layered heterostructures although their effect on work hardening was not discussed [39].

In conclusion, strain localization by shear bands seems unavoidable in nanostructures. However, in GS materials the detrimental shear bands could be harnessed to benefit ductility. Specifically, in a gradient structured specimen shear bands nucleated early in the NS and propagated along the gage length, instead of across the specimen cross-section as normally observed in homogeneous materials. This delocalized the shear bands to form an LSZ. Strain gradient was produced in the propagating front of the LSZ, which produced back-stress hardening $[14,34]$ to stabilize the propagating shear bands. In addition, strain gradient were also produced near the interfaces between the LSZ and CG central layer, which produces more back-stress hardening. Dislocation hardening capability in the LSZ was recovered after initial strain softening, which, along with back-stress hardening, induces synergistic strain hardening to help with ductility in NS layer.

\section{Impact statement}

We propose strategies for synergistic strain hardening in gradient structure by stabilizing shear band and strain gradients, turning harm of shear band into benefit of ductility.

\section{Disclosure statement}

No potential conflict of interest was reported by the authors.

\section{Funding}

This work was supported by the National Key Basic Research Program of China [grant numbers 2017YFA 0204402, 2017YFA0204403], the National Natural Science Foundation of China (NSFC) [grant numbers 11472286, 11572328, 11672313 and 11790293], and the Strategic Priority Research Program of the Chinese Academy of Sciences [grant number XDB22040503]. Y. Z. was also supported by the US Army Research Office [W911 NF-12-1-0009].

\section{References}

[1] $\mathrm{Lu} \mathrm{K}$. Stabilizing nanostructures in metals using grain and twin boundary architectures. Nature Rev Mater. 2016;1:16019.

[2] Meyers MA, Mishra A, Benson DJ. Mechanical properties of nanocrystalline materials. Prog Mater Sci. 2006;51:427-556.

[3] Ovid'ko IA, Valiev RZ, Zhu YT. Review on superior strength and enhanced ductility of metallic nanomaterilas. Prog Mater Sci. 2018;94:462-540.

[4] Valiev RZ, Estrin Y, Horita Z, et al. Fundamentals of superior properties in bulk nanoSPD materials. Mater Res Lett. 2016;4:1-21.

[5] Lu L, Chen X, Huang X, et al. Revealing the maximum strength in nanotwinned copper. Science. 2009;323:607610.

[6] Wu G, Chan K-C, Zhu LL, et al. Dual-phase nanostructuring as a route to high-strength magnesium alloys. Nature. 2017;545:80-83. 
[7] Liu XC, Zhang HW, Lu K. Strain-induced ultrahard and ultrastable nanolaminated structure in nickel. Science. 2013;342:337-340.

[8] Zhou X, Li XY, Lu K. Enhanced thermal stability of nanograined metals below a critical grain size. Science. 2018;360:526-530.

[9] Zhu YT, Liao XZ. Nanostructured metals-retaining ductility. Nature Mater. 2004;3:351-352.

[10] Wang YM, Chen MW, Zhou FH, et al. High tensile ductility in a nanostructured metal. Nature. 2002;419: 912-915.

[11] Fang TH, Li WL, Tao NR, et al. Revealing extraordinary intrinsic tensile plasticity in gradient nano-grained copper. Science. 2011;331:1587-1590.

[12] Wu XL, Jiang P, Chen L, et al. Extraordinary strain hardening by gradient structure. Proc Natl Acad Sci USA. 2014;111:7197-7201.

[13] $\mathrm{He} \mathrm{BB}, \mathrm{Hu} \mathrm{B}$, Yen HW, et al. High dislocation densityinduced large ductility in deformed and partitioned steels. Science. 2017;357:1029-1032.

[14] Wu XL, Yang MX, Yuan FP, et al. Heterogeneous lamellar structure unites ultrafine-grain strength with coarsegrain ductility. Proc Natl Acad Sci USA. 2015;112: 14501-14505.

[15] Yang MX, Yan DS, Yuan FP, et al. Dynamically reinforced heterogeneous grain structure prolongs ductility in a medium-entropy alloy with gigapascal yield strength. Proc Natl Acad Sci USA. 2018;115:72247229.

[16] Kou HN, Lu J, Li Y. High-strength and high-ductility nanostructured and amorphous metallic materials. Adv Mater. 2014;26:5518-5524.

[17] Wu XL, Yuan FP, Yang MX, et al. Nanodomained nickel unite nanocrystal strength with coarse-grain ductility. Sci Rep. 2015;5:11728.

[18] Jia D, Wang YM, Ramesh KT, et al. Deformation behavior and plastic instabilities of ultrafine-grained titanium. Appl Phys Lett. 2001;79:611-613.

[19] Chen AY, Li DF, Zhang JB, et al. Make nanostructured metal exceptionally tough by introducing non-localized fracture behaviors. Scr Mater. 2008;59:579-582.

[20] Hart EW. A theory for flow of polycrystals. Acta Metall. 1967;15:1548-1549.

[21] Weber L, Kouzeli M, San Marchi C, et al. On the use of Considere's criterion in tensile testing of materials which accumulate internal damage. Scr Mater. 1999;41: 549-551.

[22] Wei Q, Kecskes L, Jiao T, et al. Adiabatic shear banding in ultrafine-grained $\mathrm{Fe}$ processed by severe plastic deformation. Acta Mater. 2004;52:1859-1869.
[23] Bian XD, Yuan FP, Zhu YT, et al. Gradient structure produces superior dynamic shear properties. Mater Res Lett. 2017;5:501-507.

[24] Ma Y, Yuan FP, Yang MX, et al. Dynamic shear deformation of a CrCoNi medium-entropy alloy with heterogeneous grain structures. Acta Mater. 2018;148:407-418.

[25] Lu K, Lu J. Surface nanocrystallization (SNC) of metallic materials: presentation of the concept behind a new approach. J Mater Sci Technol. 1999;15:193-201.

[26] Cao Y, Ni S, Liao XZ, et al. Structural evolutions of metallic materials processed by severe plastic deformation. Mater Sci Eng R. 2018;133:1-59.

[27] Ashby MF. The deformation of plastically non-homoge neous materials. Philos Mag. 1970;21:399-424.

[28] Gao HJ, Huang Y, Nix WD, et al. Mechanism-based strain gradient plasticity - I. Theory. J Mech Phys Solids. 1999;47:1239-1263.

[29] Gao HJ, Huang YG. Geometrically necessary dislocation and size-dependent plasticity. Scr Mater. 2003;48:113118.

[30] Zeng Z, Li XY, Xu DS, et al. Gradient plasticity in gradient nano-grained metals. Extreme Mech Lett. 2016;8:213-219.

[31] Wu XL, Jiang P, Chen L, et al. Synergetic strengthening by gradient structure. Mater Res Lett. 2014;2:185-191.

[32] Li JJ, Weng GJ, Chen SH, et al. On strain hardening mechanism in gradient nanostructure. Int J Plasticity. 2017;88:89-107.

[33] Wu XL, Zhu YT. Heterogeneous materials: a new class of materials with unprecedented mechanical properties. Mater Res Lett. 2017;5:527-532.

[34] Yang MX, Pan Y, Yuan FP, et al. Back stress strengthening and strain hardening in gradient structure. Mater Res Lett. 2016;4:145-151.

[35] Liu XL, Yuan FP, Zhu YT, et al. Extraordinary Bauschinger effect in gradient structured copper. Scripta Materialia. 2018;150:57-60.

[36] Ma E, Zhu T. Towards strength-ductility synergy through the design of heterogeneous nanostructures in metals. Mater Today. 2017;20:323-331.

[37] Wilson DV. Influences of cell-walls and grain-boundaries on transient responses of an IF steel to changes in strain path. Acta Metall Mater. 1994;42:1099-1111.

[38] Ni S, Wang YB, Liao XZ, et al. Strain hardening and softening in a nanocrystalline $\mathrm{Ni}-\mathrm{Fe}$ alloy induced by severe plastic deformation. Mater Sci Eng A. 2011;528:33983403.

[39] Huang CX, Wang YF, Ma XL, et al. Interface affected zone for optimal strength and ductility in heterogeneous laminate. Mater Today. 2018;21:713-719. 